\title{
Acknowledgement of Reviewers for 2014
}

Published online: 21 October 2014

(C) Springer Science+Business Media New York 2014

The Editor of the Journal of Family Violence would like to acknowledge and thank all of the reviewers of the journal for their hard work throughout the year in reviewing articles and providing important feedback to authors. The following people provided this service during 2014, in addition to those on our editorial board:

Nor Ba'yan Abdul Kadir

Randell Alexander

Faisal Ibrahim Al-Matalka

Kathleen Basile, $\mathrm{PhD}$.

Tina Bloom

Gayle Brewer

Michael J. Brown

John Caffaro

Esther Calvete

Ricardo Carrillo

Kathleen Carty

Eduardo Horacio Cazabat

Catherine Cerulli

Alan Cohen

Cyleste Collins

Lisa Conradi

Wendy Deaton

Erika DeJonghe

Ruth DeRosa

Amber Douglas

Emily Douglas

Athena A. Drewes

Donath Emusu

Heather Foran

Mary Ann Forgey

Cindy Fraga

Donald "Rick" Froyd Jr

George Gaither

Biswajit Ghosh

Michele Harway

Brittany Hayes

Michael Hertica

Jun Sung Hong
Judy Hughes

Matthew Huss

Mohammad Reza Iravani

Kristine M. Jacquin

Linda Jeffrey

Ji Hyon Kang

Shelby Kaura

Poco D. Kernsmith

Siamak Khodarahimi

Mona Khoury-Kassabri

Jessie 1. Krienert

Shanti Kulkarni

Jessica Lambert

David M. Lawson

Kun-Hua Lee

Penny A. Leisring

Peter Lehmann

Rebecca J. Macy

Alison Marganski

Coutrney McDonald

Lynn Michalopoulos

Janet Mullings

Laura Nabors

Margaret O'Dougherty Wright

Ijeoma Nwabuzor Ogbonnaya

Sonia Oveisi

Jillian Panuzio Scott

Brian Payne

Anthony Pinizzotto

Leslee Pollina

Keevia Yvette Porter

Kimberly Randell

Evelina Rasanen
Richard Rappaport

Vaishali Raval

Ines Carvalho Relva

Lynette M. Renner

Danuta Rode

Amir Mohammad Sayem

Jeremiah Schumm

Anne Shaffer

Ryan C. Shorey

Douglas B. Smith

Roberta Sprague

Evan Stark

Ketan Tailor

Anthony Tasso

Gokce Tekin Kurt

Jeff R. Temple

Agnes Tiwari

Leslie M. Tutty

Emilio Ulloa

Kevin J. Vagi

Katayon Vakilian

Omara Rivera-Vazquez

Wendy Verhoek-Oftedahl

Jeffrey A. Walsh

Jackie White

Svetlana Yampolskaya

Hatice Yildiz

Gerald Young

Muhammad Zakar

Rubeena Zakar

Egbert Zavala 